\title{
Implementação da Política Nacional de Atenção Integral à Saúde do Homem: desafios vivenciados por enfermeiras
}

\author{
Implementation of the National Policy for Comprehensive Attention \\ to Men's Health: challenges experienced by nurses \\ Implementación de la Política Nacional de Atención Integral a la \\ Salud del Hombre: desafíos vividos por las enfermeras
}

Como citar este artigo

Sousa AR, Oliveira JA, Almeida MS, Pereira A, Almeida ES, Vergara Escobar OJ. Implementation of the National Policy for Comprehensive Attention to Men's Health: challenges experienced by nurses. Rev Esc Enferm USP. 2021;55:e03759. https://doi.org/10.1590/S1980-220X2020023603759

\section{Anderson Reis de Sousa ${ }^{1}$ \\ Josias Alves de Oliveira ${ }^{1}$ \\ Marcio Soares de Almeida ${ }^{1}$ \\ Álvaro Pereira ${ }^{1}$ \\ Éric Santos Almeida ${ }^{2}$ \\ Oscar Javier Vergara Escobar ${ }^{3}$}

${ }^{1}$ Universidade Federal da Bahia, Escola de Enfermagem, Programa de Pós-Graduação em Enfermagem, Salvador, BA, Brasil.

${ }^{2}$ Faculdade Pitágoras de Eunápolis, Eunápolis, BA, Brasil.

${ }^{3}$ Fundación Universitaria Juan N Corpas, Bogotá, Colômbia.

\begin{abstract}
Objective: To analyze the challenges experienced by nurses in the implementation of the National Policy for Comprehensive Attention to Men's Health. Method: Descriptive, qualitative study, carried out with nurses working in Primary Health Care in a city in the state of Bahia, Brazil. Individual interviews were carried out and then analyzed using the Discourse of the Collective Subject, in the light of the guidelines of the National Policy for Comprehensive Attention to Men's Health. Results: A total of 40 nurses participated. The challenges for the implementation of the policy are related to the inoperability of government actions, weaknesses in municipal management, underfunding and discontinuity of actions. Conclusion: Nurses face complex macro-management challenges in the implementation of the National Policy for Comprehensive Attention to Men's Health in Primary Health Care.
\end{abstract}

\section{DESCRIPTORS}

Nursing; Men's Health; Health Policy; Primary Health Care; Health Care (Public Health). 


\section{INTRODUÇÃO}

A saúde do homem tornou-se uma preocupação mundial $^{(1-3)}$. Dados do Brasil, em 2016, apontavam que as mulheres viviam, em média, 7,4 anos a mais que os homens e tinham uma expectativa de vida em torno de 78,7 anos - para os homens, essa expectativa era de 71,3 anos. Além disso, observava-se que os indicadores de morbimortalidade eram mais elevados na parcela masculina da população, com destaque para as causas externas ${ }^{(4-5)}$.

Diante disso, o Ministério da Saúde no Brasil implantou, no ano de 2009, a Política Nacional de Atenção Integral à Saúde do Homem (PNAISH), a qual elegeu a Atenção Primária à Saúde (APS) como cenário fundamental de promoção do cuidado aos homens. Nesse contexto, o fomento de incentivos orçamentários e o envolvimento do público masculino nas ações de saúde focalizaram a possibilidade de uma transformação do panorama brasileiro da saúde dos homens ${ }^{(6-7)}$.

A PNAISH tem como objetivo principal a promoção de ações na perspectiva de garantir a ampliação do acesso dos homens aos serviços de saúde, em especial à APS, considerando os diferentes arranjos organizacionais, a capacidade operacional e tecnológica das redes de serviços que compõem os sistemas locais de saúde. Essa política assumiu a compreensão das singularidades e especificidades masculinas sob o olhar para as masculinidades em seus diversos contextos socioculturais, a qual tem como diretrizes centrais o entendimento da saúde do homem como um conjunto de ações direcionadas à promoção, prevenção, assistência e recuperação em todo o território nacional ${ }^{(8)}$.

Nesse contexto, em que a APS se configura como porta de entrada preferencial do Sistema Único de Saúde (SUS), a inserção da enfermeira através das equipes multidisciplinares assume singular importância, pois sua atuação promovendo práticas que articulam assistência clínica e gerência administrativa dos serviços contribui para a produção cotidiana do cuidado. São essas profissionais que por vezes atuam como líderes das equipes, sendo responsáveis por ações de coordenação, supervisão, monitoramento, educação/formação continuada e pesquisa, contribuindo para a implantação e implementação das ações pretendidas pela PNAISH em todos os níveis, sejam centrais ou municipalizados ${ }^{(9)}$.

Frente a esse cenário, pode-se observar que a atuação ampla da enfermeira na APS é essencial para a implementação de políticas públicas de saúde, dado que o seu exercício profissional nesse contexto integra a gestão de recursos, a articulação de processos administrativos, as ações de ensino-aprendizagem, a execução da educação permanente e continuada relacionada à saúde masculina, além de atuar diretamente na assistência à população em todo o seu ciclo de vida, materializada em consultas individuais e intervenções coletivas. Destarte, competências e habilidades podem ser facilmente identificadas no perfil profissional de enfermeiras que atuam na APS, como a tomada de decisão, o acolhimento, a promoção de vínculo e responsabilização, a flexibilidade, a criatividade, a obtenção do conhecimento científico, a expertise clínica, o compromisso e a capacidade de negociação, o que aporta expressiva contribuição para o avanço da PNAISH no Brasil ${ }^{(10)}$.
Dessa maneira, quando bem posicionadas e com condições favoráveis para atuar, as enfermeiras utilizam da visão estratégica e mobilização para a concretização do trabalho em equipe, o que poderá facilitar a operacionalização de ações que visem à gestão/produção do cuidado aos usurários do serviço, notoriamente, foco na Estratégia de Saúde da Família $(\mathrm{ESF})^{(10)}$.

Todavia, ao analisar o contexto e o processo saúde-doença da população masculina, observa-se a necessidade de inclusão desse público nas ações estratégicas de promoção à saúde e prevenção de doenças e agravos, considerando suas especificidades, o que exige dos profissionais de saúde um olhar amplo direcionado para a integralidade. Contudo, atuações relacionadas à saúde dos homens ainda são pouco estruturadas, o que pode culminar em dificuldades na implementação de ações dedicadas a esse público, tal como é a implementação de uma política de saúde, no cotidiano do trabalho em saúde ${ }^{(6)}$.

Dessa maneira, a PNAISH pode ser vista como proposta estratégica que visa aproximar o público masculino e inseri-lo nos cuidados preventivos de saúde. Porém, cabe ressaltar as dificuldades encontradas pelos profissionais que atuam na APS, para que a política possa ser de fato executada. Diversos fatores permeiam a implementação da política, como o planejamento na macrogestão e a transposição para a operacionalização das atividades de assistência na microgestão, o que pode gerar desafios para a prática profissional em Enfermagem ${ }^{(9,11-12)}$. Além disso, um cenário desfavorável tem sido identificado no Brasil pelo próprio Ministério da Saúde, quando se diz respeito aos avanços na implementação da política no país, que envolve, entre outros fatores, as questões de gênero, as quais influenciam a relação dos homens com os serviços de saúde ${ }^{(9,12)}$.

Ainda que esse panorama tenha sido apresentado, há lacunas no conhecimento científico da disciplina de Enfermagem no tocante à atenção à saúde e à produção do cuidado direcionado ao público masculino. No âmbito da implementação da PNAISH, estudos originais são limitados, o que justifica a emergência deste estudo e o torna relevante para a formação e prática de Enfermagem.

Ante o exposto, este estudo foi guiado pela seguinte questão de pesquisa: Quais os desafios vivenciados por enfermeiras na implementação da PNAISH? Este artigo teve o objetivo de analisar os desafios vivenciados por enfermeiras na implementação da PNAISH.

\section{MÉTODO}

\section{TIPO DE ESTUdO E CENÁRIO}

Trata-se de um estudo brasileiro, descritivo, qualitativo, realizado com 40 enfermeiras que atuam na APS, especificamente na ESF, sendo sete Unidades Básicas de Saúde (UBS) e 26 Unidades de Saúde da Família (USF), em um município da Bahia, Brasil.

\section{CRITÉRIOS de SELEÇ̃̃o}

Foram incluídas na pesquisa enfermeiras que atuavam prestando assistência direta aos usuários. Não se incluiu na pesquisa enfermeiras que estavam em treinamento nas 
unidades, que se encontravam retirando férias profissionais e estagiárias de formação curricular em Enfermagem. No total da amostra, quatro profissionais se recusaram a participar da pesquisa: duas argumentando não ter conhecimento e aproximação com a temática, uma por levantar ausência de tempo para responder aos questionamentos e a última profissional não quis revelar o motivo da recusa.

\section{Coleta de Dados}

Para coleta de dados, realizou-se um levantamento das unidades de saúde existentes no município, mediante articulação junto à Secretaria Municipal de Saúde, por meio da Divisão de Enfermagem, que ocorreu entre os meses de agosto e dezembro do ano de 2017 e, posteriormente, entre março e junho de 2018. Após esse momento, realizou-se entrevista individual em profundidade, guiada por um instrumento semiestruturado composto por questões fechadas, que versavam sobre as características profissionais, sociais e econômicas, e questões abertas sobre a problemática central do estudo: Conte-nos a sua vivência profissional face a atenção à saúde de homens? Fale-nos como tem se dado a implementação da PNAISH em seu território? Um teste piloto foi aplicado previamente no grupo de pesquisa e depois junto à três participantes. Os dados apreendidos não foram incorporados à amostra deste estudo, que não constou de entrevistas repetidas.

As entrevistas foram gravadas sob a utilização de um gravador profissional, posteriormente transcritas na íntegra e apresentadas às participantes do estudo para aquisição da anuência, conforme as diretrizes propostas pelo Consolidated criteria for reporting qualitative research (COREQ), que norteou todo o estudo. Ademais, o recrutamento das participantes deu-se de modo consecutivo, a partir de uma seleção intencional. Faz-se saber que os pesquisadores do estudo possuíam aproximação prévia com o objeto do estudo. Um deles, o pesquisador responsável, do sexo masculino e mestre em Enfermagem, exercia atividade profissional como docente e realizava ações de educação em saúde nos serviços e na formação de recursos humanos e acadêmicos na área. Os demais membros da equipe que realizaram a pesquisa possuíam graduação em Enfermagem, ambos com formação em pesquisa e treinamento quanto ao método empregado.

As entrevistas ocorreram em ambiente reservado, nas instalações das unidades de saúde, em horários em que as enfermeiras não estavam prestando atendimento aos usuários(as), conforme agendamento prévio. As participantes foram abordadas face a face. Apenas os pesquisadores e a participante se encontravam presentes no momento da entrevista. O tempo de duração médio das entrevistas foi de 60 minutos. Notas de campo foram realizadas após as entrevistas, mas não foram incorporadas como material empírico para análise. $\mathrm{O}$ processo de coleta só foi iniciado após visita prévia às unidades para apresentação da pesquisa, e posteriormente à realização do convite e assinatura do Termo de Consentimento Livre e Esclarecido (TCLE).

A composição do corpus empírico deu-se pelo emprego da amostragem teórica derivada dos dados. Foram levadas em consideração as distintas características territoriais vivenciadas pelas enfermeiras, o que conduziu os pesquisadores a aprofundarem as investigações na busca por evidenciar as coocorrências, convergências e complementariedades nos discursos das profissionais. Dos dados derivados, 10 códigos teóricos foram analisados. Destaca-se que as participantes tiveram acesso às entrevistas transcritas, opinaram sobre e tiveram acesso à apresentação sobre os resultados em um evento temático realizado pela secretaria municipal de saúde.

\section{ANÁLISE E TRATAMENTO DOS DADOS}

Os dados coletados foram organizados, sistematizados e processados no Software NVIVO10 para análise qualitativa. A amostra é constituída de dados sociodemográficos, profissionais e empíricos relativos ao objeto de investigação central do estudo. A análise foi orientada pelo método do Discurso do Sujeito Coletivo (DSC), que resgata constructos estruturantes das Representações Sociais, demarcado pelo reconhecimento representativo na dimensão individual, em articulação à sua dimensão coletiva. Ambas as dimensões são agrupadas em categorias semânticas gerais, associando os conteúdos das opiniões de sentido semelhante presentes nos diferentes depoimentos ${ }^{(13)}$. As participantes foram identificadas através da descrição própria do método: "DSC de enfermeiras que atuam na ESF".

Desse modo, o DSC, através de suas figuras metodológicas denominadas de Expressões Chaves, Ideias Centrais e Ancoragens, possibilita a formação de discursos sínteses, capazes de representar uma coletividade na pessoa de um indivíduo ${ }^{(13)}$. Os achados foram analisados sob o prisma do referencial teórico/normativo da PNAISH em seus princípios e diretrizes ${ }^{(8)}$. Os dados mostraram-se alinhados às conclusões, convergindo-se para o pressuposto do estudo e a problematização dos achados discursivos evidenciados.

\section{Aspectos éticos}

O estudo esteve vinculado ao projeto de pesquisa-matriz, intitulado "Atenção à saúde de homens em um cenário do Nordeste brasileiro", aprovado pelo Comitê de Ética em Pesquisa (CEP), sob o parecer de número 1.208.304, junto ao subprojeto de pesquisa, intitulado "Enfermagem na atenção à saúde de homens: desafios de implementação das ações na Estratégia de Saúde da Família”, também aprovado pelo CEP, sob o parecer de número 996.821, ambos geridos com financiamento próprio. Foram preservadas, em todo o processo de desenvolvimento da pesquisa, as recomendações éticas para realização de estudos envolvendo seres humanos, atendendo as diretrizes da Resolução 466 de 2012 do Conselho Nacional de Saúde, assim como a Resolução n ${ }^{\circ}$ 580 de 22 de março de 2018, a qual regulamenta o disposto no item XIII.4 da Resolução do Conselho Nacional de Saúde (CNS) no 466, além de estabelecer particularidades éticas das pesquisas com seres humanos em instituições do SUS. Para tanto, manteve-se o direito à autonomia na participação no estudo, o sigilo das informações, o anonimato das depoentes, sendo descritas conforme a apresentação metodológica do DSC, identificadas como DSC de enfermeiras que atuam na ESF. 


\section{RESULTADOS}

As participantes do estudo foram em sua totalidade do sexo feminino, com identidade de gênero cisgênera, heterossexuais, casadas, de raça/cor autorreferida parda, com faixa etária entre 25 e 48 anos. Atuavam nas unidades de saúde há mais de um ano, com tempo total de formação entre $2 \mathrm{e}$ 16 anos. Quanto à formação acadêmica da pós-Graduação, as enfermeiras eram especialistas na área de saúde pública e/ou saúde da família, seguida de atenção às urgências e emergências. A maioria possuía um vínculo empregatício.

O discurso do sujeito coletivo das enfermeiras revelou que os desafios para a implementação da PNAISH estão concentrados na inoperância das ações governamentais, na presença de fragilidades da gestão municipal, no subfinanciamento e, por consequência, na descontinuidade das ações.

A inoperância das ações governamentais a nível federal, que gera desafios para a implementação da política, é desvelada no discurso das enfermeiras, destacando-se a limitação das estratégias de ampliação do acesso de homens aos serviços de saúde: o fortalecimento do estímulo e a formação profissional para atendimento das demandas masculinas, a ausência de protocolos de orientação ministerial, a baixa divulgação e o descompromisso e não priorização por parte dos governantes para com a atenção à saúde masculina. Os achados serão apresentados nos Quadros 1, 2, 3 e 4 abaixo.

Quadro 1 - Discurso do Sujeito Coletivo das enfermeiras que atuavam na Estratégia de Saúde da Família sobre a inoperância das ações governamentais, Bahia, Brasil, 2020.

\begin{tabular}{|c|c|}
\hline $\begin{array}{l}\text {...] Acesso do } \\
\text { de captação... } \\
\text {....] }\end{array}$ & $\begin{array}{l}\text { A política de saúde do homem é positiva, pois busca abordar os problemas que atingem especificamente a } \\
\text { população masculina, como proposta de promoção da saúde e a prevenção das doenças. No entanto, as } \\
\text { suas estratégias necessitam ser melhoradas a fim de facilitar o acesso dos homens na busca pelos serviços }\end{array}$ \\
\hline $\begin{array}{l}\text { Ideias cen } \\
\text { ações... di } \\
\text { que nos o }\end{array}$ & $\begin{array}{l}\text { Ie os protıssıonaıs de saude desenvolvam estrateggas de captaçao dos homens, n } \\
\text { senvolvimento de consultas e ações de promoção específicas para o público ma }\end{array}$ \\
\hline Ancoragem: & ortalecimento para que haja a prese \\
\hline
\end{tabular}

Fonte: Dados da pesquisa.

As fragilidades na gestão municipal emergiram no discurso coletivo como desafio enfrentado para a implementação da PNAISH, contrapondo a disposição e atuação das enfermeiras que reconhecem as demandas masculinas advindas do território às práticas da gestão municipal, que estabelece metas a serem atingidas, mas não garante condições e recursos para sua contemplação. Destaca-se, ainda, a ausência de infraestrutura, como a falta de veículos para locomoção das equipes e o desenvolvimento das atividades fora da unidade.

Quadro 2 - Discurso do Sujeito Coletivo de enfermeiras que atuam na Estratégia de Saúde da Família sobre as fragilidades na gestão municipal, Bahia, Brasil, 2020.

\begin{tabular}{|c|c|}
\hline & \multirow{3}{*}{$\begin{array}{l}\text { Eu estou diretamente em atendimento na unidade de saúde, conheço as necessidades que afligem a } \\
\text { população e as demandas da área, mas os gestores que estão no topo, sejam municipais como o secretário } \\
\text { de saúde ou coordenadores, só mandam as atribuições para serem cumpridas, implantam e cobram, mas } \\
\text { não asseguram que as ações sejam efetivadas. Não dão o suporte necessário no que precisamos para } \\
\text { termos êxito nas ações e nem acompanham como estas podem ser implementadas. Além disso, não se } \\
\text { preocupam em estar junto com a equipe de saúde para promover essa política e ver de perto a necessidade } \\
\text { que o homem tem, o que pode facilitar a melhoria do acesso e a assistência. Não tenho estrutura adequada } \\
\text { para desenvolver as ações da política, como as ações educativas, a locomoção com a disponibilização de } \\
\text { transporte da equipe até a área de abrangência na comunidade, ter que utilizar o meu próprio veículo e me } \\
\text { expor a perigos, devido às áreas de risco no meu território (DSC, enfermeiras que atuam na ESF). }\end{array}$} \\
\hline & \\
\hline & \\
\hline
\end{tabular}

Fonte: Dados da pesquisa.

O subfinanciamento do sistema público de saúde foi apontado pelas enfermeiras como importante desafio para a implementação da PNAISH. A atuação dessas profissionais no âmbito da ESF apresenta pouca governabilidade sobre a alocação e a distribuição dos recursos financeiros necessários para garantir a atenção à saúde dos homens, o que compromete a realização de ações, a divulgação e até mesmo os deslocamentos necessários para atividades no território de abrangência dos serviços.

Quadro 3 - Discurso do Sujeito Coletivo de enfermeiras que atuam na Estratégia de Saúde da Família sobre o subfianciamento para a implementação da PNAISH, Bahia, Brasil, 2020.

\begin{tabular}{|c|c|}
\hline $\begin{array}{l}\text { Expressões-chave: [...] recursos financeiros... } \\
\text { transporte... investimento... campanhas [...]. }\end{array}$ & \multirow{3}{*}{$\begin{array}{l}\text { É preciso que o governo invista recursos financeiros para atrair os homens para a unidade de saúde. } \\
\text { Essa é uma responsabilidade do gestor em demandá-los, pois eu, enquanto enfermeira, não tenho } \\
\text { essa competência. Faltam recursos para realizar as ações de educação em saúde, divulgação dos } \\
\text { serviços, campanhas de saúde nas redes sociais e nos programas de rádio. Sem esses benefícios, o } \\
\text { desenvolvimento de ações constantes sobre a conscientização do cuidado à saúde se torna difícil } \\
\text { (DSC, enfermeiras que atuam na ESF). }\end{array}$} \\
\hline Ideias centrais: $[\ldots]$ falta de recursos $[\ldots]$. & \\
\hline $\begin{array}{l}\text { Ancoragem: }[. . .] \text { eu, enquanto enfermeira, } \\
\text { não tenho este potencial... já que não somos } \\
\text { beneficiados, nesta unidade [...]. }\end{array}$ & \\
\hline
\end{tabular}

Fonte: Dados da pesquisa. 
A descontinuidade das ações foi observada enquanto fator desafiador para as enfermeiras. O discurso emergido aponta que a PNAISH não tem sido priorizada pelos governantes, sendo esquecida, o que impacta diretamente na continuidade das ações, fazendo com que estas deixem de acontecer no cotidiano dos serviços de saúde. As enfermeiras relatam que o processo de implementação não é acompanhado, nem monitorado, não sendo conferido o apoio necessário para tal. Além disso, o discurso enfatiza que as ações destinadas à saúde de homens limitam-se à prevenção e ao tratamento do câncer de próstata, por meio das ações de rastreamento, realizadas na campanha intitulada de "Novembro Azul". Porém, não são medidas estendidas para outros contextos e demais períodos do ano.

Quadro 4 - Discurso do Sujeito Coletivo de enfermeiras que atuam na Estratégia de Saúde da Família sobre a descontinuidade das ações para a implementação da PNAISH, Bahia, Brasil, 2020.

\begin{tabular}{|c|c|}
\hline $\begin{array}{l}\text { Expressões-chave: }[\ldots] \text { negativo... } \\
\text { não se dá prioridade [...]. }\end{array}$ & $\begin{array}{l}\text { As políticas de saúde são implantadas, mas, a depender do público-alvo que a essa se destina, o governo não } \\
\text { direciona a mesma prioridade, o que se torna negativo, pois as ações perdem a continuidade, são esquecidas. }\end{array}$ \\
\hline $\begin{array}{l}\text { Ideias centrais: }[. . .] \text { quando estamos } \\
\text { no Novembro Azul, o governo } \\
\text { disponibiliza tudo [...]. } \\
{[\ldots] \text { as ações precisam acontecer o }} \\
\text { ano inteiro [...]. }\end{array}$ & $\begin{array}{l}\text { Esta é uma realidade que eu observo para com a PNAISH. Não se deve apenas implantar, mas os governantes e } \\
\text { gestores devem cobrar para que a política seja implementada. Devem participar e acompanhar de fato, garantir } \\
\text { o suporte necessário, pois se isto não acontecer como que a política vai funcionar? Só quando estamos na } \\
\text { campanha do Novembro Azul o governo disponibiliza tudo, como a realizaça de exames, rastreamento, vagas } \\
\text { para consultas médicas, encaminhamento para as unidades especializadas e suporte adequado às demandas, mas } \\
\text { nos outros dias os homens precisam marcar, esperar pela regulação médica para realizar o encaminhamento caso }\end{array}$ \\
\hline $\begin{array}{l}\text { Ancoragem: }[\ldots] \text { perdendo a } \\
\text { continuidade... sendo esquecidas }[\ldots] \text {. }\end{array}$ & $\begin{array}{l}\text { haja algum problema maior, como a identificação de um câncer. E tudo isso leva muito tempo. As ações precisam } \\
\text { acontecer o ano inteiro e não apenas em um mês (DSC, enfermeiras que atuam na ESF). }\end{array}$ \\
\hline
\end{tabular}

Fonte: Dados da pesquisa.

\section{DISCUSSÃO}

As limitações deste estudo concentraram-se na aplicação de uma única técnica para a produção dos dados empíricos, o que pode ter refletido nos achados que foram apreendidos em contraste à combinação de diferentes métodos. Ademais, outros profissionais da Enfermagem que atuam no âmbito da $\mathrm{ESF}$, como as técnicas e auxiliares de enfermagem, não foram inseridos, implicando nuances ainda a serem exploradas em estudos futuros. Por fim, o envolvimento de profissionais de Enfermagem que atuavam no âmbito específico da gestão e área técnica em saúde dos homens poderia desvelar outros aspectos que complementariam os dados apresentados.

Neste estudo, os achados são capazes de apresentar analiticamente as dimensões dos desafios vivenciados por enfermeiras no cotidiano das suas práticas profissionais na APS no que concerne à implementação da PNAISH. O discurso das enfermeiras revela os entraves na promoção das práticas de cuidado que alcance e envolva os homens no âmbito da APS, apontando para os desafios para a implementação da PNAISH. Além disso, expõe a direcionalidade que a política assume, como promotora do acesso, num cenário que ainda se traduz insuficiente para a transformação das complexas questões que são demandadas por esse público, no contexto da atenção à saúde e produção do cuidado, por transpassarem aspectos da construção social das masculinidades.

No que tange às dificuldades de inserção dos homens na perspectiva da produção do cuidado, caracterizam-se três elementos contextuais: aquele referente aos próprios homens, baseado na construção de suas masculinidades; outro relacionado à dimensão profissional, marcado pela baixa capacidade em transpor os princípios da política em ações; e, por último, a natureza organizacional dos serviços, relacionada à demarcação generificada das ações e dos serviços de saúde, conforme já corroborado por outro estudo ${ }^{(14)}$.

Além disso, considerando a política como instrumento normatizador necessário, sua concretude não se faz suficiente para transformar a realidade, pois, simbolicamente, a singularidade do substantivo "homem" contido no título do documento reflete o não reconhecimento da diversidade de construção e exercício das masculinidades, contrapondo ao corpo do texto que explicita outra compreensão em sua diretriz $^{(7)}$. Outra diretriz da PNAISH é a integração das entidades da sociedade civil organizada na corresponsabilização das ações governamentais, considerando a saúde como meio de exercício da cidadania ${ }^{(8)}$. Sobretudo, os achados deste estudo evidenciam as invisibilidades dessa integração social nas ações em saúde, que impactam também o reconhecimento social da relevância dessa política de saúde.

Embora a relevância da política seja reconhecida, as enfermeiras apontaram para as limitações das ações da gestão, que influenciam no processo de trabalho. Nota-se no discurso a inexistência de vontade política para a implementação da PNAISH, o que demanda esforços de diferentes ordens, considerando os aspectos estruturais e os custos que essa política possui.

Ademais, outro estudo, ao analisar a implementação da PNAISH em uma capital brasileira, revelou que o processo de gestão é fragmentado e caracterizado pela instabilidade dos atores envolvidos na condução dessa política, com proposição de ações inespecíficas, sem justificativas epidemiológicas ou planejadas sem a adequada apreensão da realidade local e diagnóstico situacional, o que se refletia na incapacidade de transformação do cenário de saúde da população masculina ${ }^{(15)}$. Também é válido destacar a observação da inexistência de um instrumento que possibilite à enfermeira orientar o cuidado à saúde dos homens no âmbito da ESF, o que fragiliza o cuidado destinado a esse público. Outro estudo ressalta a falta de qualificação profissional para atender às demandas vinculadas à saúde do homem, o que pode contribuir para a baixa procura aos serviços de APS ${ }^{(16)}$.

Diante disso, ao considerar a morbimortalidade masculina, muitos problemas poderiam ser evitados se os homens realizassem consultas com profissionais de saúde de maneira periódica e adotassem medidas de cuidado à saúde preventiva, como o emprego de atitudes e hábitos saudáveis. Dessa 
maneira, é notório que tal distanciamento masculino dessas práticas tem aumentado significativamente os custos para o SUS, bem como sobrecarrega emocional e fisicamente o próprio usuário e seus familiares ${ }^{(17-20)}$.

A despeito disso, tem-se notado a importância do profissional de Enfermagem como indispensável para o atendimento ao público masculino, pois realiza atividade de cunho educativo, de prevenção e promoção à saúde, com a possibilidade de sintetizar a magnitude das ações, bem como a sua funcionalidade e aplicação prática, na tentativa de melhorar os serviços públicos de saúde ${ }^{(9)}$. Contudo, as enfermeiras apontaram que há escassez de recursos financeiros para a operacionalização das ações planejadas e sinalizaram a precarização existente no desempenho de suas funções profissionais, a exemplo da falta de veículos próprios dos serviços de saúde em que atuam, para locomoção das equipes.

Essa ausência de recurso financeiro específico tem sido um fator determinante para a descontinuidade das ações e o comprometimento do processo de implementação da política de saúde do homem. Além disso, consideraram que essa justificativa refletia uma compreensão inadequada acerca da própria natureza do sistema de saúde, no que tange à atenção básica, visto que possui alocação de recursos que poderiam viabilizar a efetiva ampliação do acesso e da promoção do cuidado masculino como proposto nas diretrizes ${ }^{(15)}$. Nesse sentido, as repercussões do subfinanciamento dos serviços de saúde e as inadequadas estratégias de políticas e ações públicas destinadas ao público masculino têm sido traduzidas nas disparidades dos indicadores de morbimortalidade entre homens e mulheres no Brasil, que chega a ser quatro vezes maior ${ }^{(21)}$.

O modelo de gestão municipal, mediante a apresentação discursiva das enfermeiras, mostrou-se verticalizado, centrado no alcance dos indicadores quantitativos numérico, pouco participativo e construtivo, revelando uma atuação distante por parte dos gestores e coordenadores. Ao caracterizar a atenção ofertada à saúde dos homens pela ESF em diferentes regiões brasileiras, achados vêm demonstrando a existência de certa incompatibilidade entre as expectativas dos gestores sobre os motivos masculinos para procura dos serviços e as reais demandas expressas por essa parcela da população, o que pode refletir uma lacuna fundamental entre os serviços e os usuários, traduzindo-se na debilidade do envolvimento desses sujeitos nas diferentes ações e práticas de cuidado oferecidas nesses espaços ${ }^{(22-23)}$.

Contudo, a partir desse cenário, é passível de questionamentos o próprio potencial que as equipes de saúde possuem para gerir a sua micropolítica, presente em seus espaços de atuação, lançando mão de ferramentas de gestão do processo de trabalho, a partir da ampliação e do fortalecimento de dispositivos como o acolhimento, vínculo, responsabilização, territorialização, matriciamento, planejamento estratégico situacional, dentre outros. O potencial do vínculo e do acolhimento na reorientação das práticas de saúde e inserção dos homens como sujeitos de direitos e cuidado tem sido evidenciado no campo de prática profissional em Enfermagem, ressaltando a capacidade desses dispositivos em viabilizar a apreensão enquanto valor de uso dos produtos do trabalho dos profissionais de saúde, que atuam em serviços que habitualmente pouco atraem os homens, como a atenção básica, na medida em que suprem as suas necessidades de saúde ${ }^{(24-25)}$.

Torna-se necessário (re)pensar politicamente a saúde de homem e a participação da Enfermagem de forma mais abrangente, de modo a considerar a produção do sujeito a partir da problematização de sua condição de saúde, instrumentalizando uma ação social, mediante as circunstâncias que os atravessam, assim como também vulnerabilizam, buscando romper com as condições impeditivas no exercício de uma masculinidade saudável e menos prejudicial e tóxica ${ }^{(2)}$.

No que concerne à descontinuidade das ações, é possível observar um discurso que revela diferentes nuances, refletindo uma prática profissional de Enfermagem ainda restritiva à lógica de operacionalização de programas de saúde. Tal problemática repercute em barreiras no aperfeiçoamento dos sistemas de informação, capazes de monitorar e/ou direcionar racionalmente as ações de saúde dos homens no Brasil, outra diretriz que consta na PNAISH ${ }^{(8,26)}$.

Sob esse desafio, destaca-se, ainda, a restrição do cuidado masculino a ações pontuais e campanhistas, como o Novembro Azul, dedicado ao rastreamento do câncer de próstata. Estudiosos analisaram as ações do "Novembro Azul" e evidenciaram as disputas subjacentes a essa campanha, escancarando o papel e a contribuição da prática médica na conformação de um modelo de cuidado à saúde do homem reduzido às ações esporádicas e que assumem caráter meramente curativista, sem contribuir efetivamente para a integralidade da saúde dos homens ${ }^{(27-29)}$.

Importa também destacar a ponderação dos dados revelados no discurso em relação às questões relacionais de gênero, haja vista que são apreendidos por mulheres, as enfermeiras, que a partir das suas construções moldam o seu olhar sobre os homens e o cuidado à saúde a ser ofertado ${ }^{(30)}$. Destaca-se, ainda, a relevância da categoria gênero no que diz respeito à pratica a ser desempenhada por enfermeiras no tocante à produção do cuidado à saúde de homens, necessitando ser, portanto, incorporada ${ }^{(7)}$.

Há necessidades de avanços quanto à perspectiva relacional de gênero e as masculinidades junto a outros marcadores estruturais que se interseccionam e desempenham influências na organização e no planejamento do cuidado em saúde. Desse modo, pode-se repensar a maneira como os serviços e os profissionais, em especial os da Enfermagem, estão organizados para alcançar os usuários, assumindo, nesse caso, os homens como centralidade na produção do cuidado. Destarte, o cenário político de austeridade fiscal põe em risco o direito à saúde, por comprometer o já precário acesso dos homens aos serviços de saúde, considerando que a escassez de recursos inviabiliza a ampliação da oferta de serviços, a continuidade do cuidado e a garantia da integralidade da atenção à saúde, o que por si só amplifica o quadro de vulnerabilidades que esses sujeitos já estão expostos em seus modos de gerir a própria vida.

\section{CONCLUSÃO}

$\mathrm{O}$ discurso coletivo das enfermeiras apontou para uma miríade de obstáculos que se impõe na garantia do direito 
à saúde para homens, implicando desafios no âmbito da concretização da PNAISH, pois se referem aos aspectos operacionais da própria política no sistema de saúde, quer seja pela falta de compromisso do Estado na promoção da saúde de homens como diretriz de organização do cuidado, quer seja pela capacidade técnica nos diferentes âmbitos da gestão do SUS ou mesmo na indisponibilidade e má alocação de recursos financeiros para a implementação e consolidação de práticas e serviços capazes de acolher e responder adequadamente às necessidades de saúde da população masculina.
Ademais, é imprescindível considerar que, além da necessidade de superação dos desafios assinalados, a efetivação da PNAISH e a promoção da saúde de homens envolvem o repensar das práticas convencionais de cuidado, outrora ainda medicalizantes, restritas ao ambiente do interior das unidades e serviços tradicionais de saúde. Nesse sentido, a própria política constitui-se como dispositivo capaz de dar visibilidade e produzir encontros que sugestivamente sejam afetivos, efetivos e transformadores dos sujeitos, sejam eles quem forem, estejam onde estiverem.

\section{RESUMO}

Objetivo: Analisar os desafios vivenciados por enfermeiras na implementação da Política Nacional de Atenção Integral à Saúde do Homem. Método: Estudo descritivo, qualitativo, realizado com enfermeiras que atuam na Atenção Primária à Saúde em um município da Bahia, Brasil. Realizou-se entrevista individual, analisada pelo método do Discurso do Sujeito Coletivo, à luz das diretrizes da Política Nacional de Atenção Integral à Saúde do Homem. Resultados: Participaram 40 enfermeiras. Os desafios para a implementação da política concentram-se na inoperância das ações governamentais, fragilidades da gestão municipal, subfinanciamento e descontinuidade das ações. Conclusão: As enfermeiras enfrentam desafios complexos de macrogestão na implementação da Política Nacional de Atenção Integral à Saúde do Homem na Atenção Primária à Saúde.

\section{DESCRITORES}

Enfermagem; Saúde do Homem; Política de Saúde; Atenção Primária à Saúde; Atenção à Saúde.

\section{RESUMEN}

Objetivo: Analizar los desafíos vividos por las enfermeras en la implementación de la Política Nacional de Atención Integral a la Salud del Hombre. Método: Se trata de un estudio descriptivo y cualitativo realizado con enfermeras que trabajan en la Atención Primaria de Salud en un municipio de Bahía, Brasil. Se llevaron a cabo entrevistas individuales y se analizaron con el método del Discurso del Sujeto Colectivo, a la luz de los lineamientos de la Política Nacional de Atención Integral a la Salud del Hombre. Resultados: Participaron 40 enfermeras. Los retos para la aplicación de la política se concentran en la inoperancia de las acciones gubernamentales, la debilidad de la gestión municipal, la infrafinanciación y la discontinuidad de las acciones. Conclusión: Las enfermeras se enfrentan a complejos retos de macrogestión para implementar la Política Nacional de Atención Integral a la Salud del Hombre en la Atención Primaria Sanitaria.

\section{DESCRIPTORES}

Enfermería; Salud del Hombre; Política de Salud; Atención Primaria de Salud; Atención a la Salud.

\section{REFERÊNCIAS}

1. National Men's Health Action Plan. Healthy Ireland-Men HI-M 2017-2021: working with men in Ireland to achieve optimum health and wellbeing [Internet]; 2016 [cited 2020 Apr 16]. Available from: https://www.mhfi.org/HI-M.pdf

2. O'Brien AP, Hurley J, Linsley P, McNeil KA, Fletcher R, Aitken JR. Men's preconception health: a primary health-care viewpoint. Am J Men Health. 2018;12(5):1575-81. https://doi.org/10.1177/1557988318776513

3. Beltrán-Sánchez H, Finch CE, Crimmins EM. Twentieth century surge of excess adult male mortality. Proc Natl Acad Sci USA. 2015;112(29):8993-8. https://doi.org/10.1073/pnas.1421942112

4. Mourão SL, Tapety FI, Monteiro CF, Feitosa LG, Lago EC. Práticas educativas à saúde do homem: desafios na Estratégia Saúde da Família. Nursing. 2019;22(251):2893-7. https://doi.org/10.36489/nursing.2019v22i251p2893-2897

5. Oliveira JC, Paula Correa ÁC, Arruda L, Mozer IT, Medeiros RM. Epidemiological profile of male mortality: contributions to nursing. Cogitare Enfermagem. 2017;22(2):e49724. https://doi.org/10.5380/ce.v22i2.49742

6. Santos EM, Figueiredo GA, Mafra AL, Reis HF, Louzado JA, Santos GM. [“Men are very tough": Men's Health in the perceptions of Family Health Strategy nurses]. Rev. APS. 2017;20(2):231-8. Portuguese. https://doi.org/10.34019/1809-8363.2017.v20.16058

7. Pereira J, Klein C, Meyer DE. [PNAISH: an analysis of its educative dimension from the gender perspective]. Saúde Soc. 2019;28(2):132-46. Portuguese https://doi.org/10.1590/s0104-12902019170836

8. Brasil. Ministério da Saúde. Política Nacional de Atenção Integral à saúde do homem. Brasília, DF: Ministério da Saúde; 2009.

9. Alves BM, Araújo CJ, Almeida SL, Guimarãoes AL. Basic care nurse's activities in connection with difficulties for the implementation of men's health policy. Rev Enferm UFPE On Line. 2017;12(11):5391-401. https://doi.org/10.5205/1981-8963-v11i12a110143p5391-5401-2017

10. Peruzzo HE, Bega AG, Lopes AP, Haddad MC, Peres AM, Marcon SS. The challenges of teamwork in the family health strategy. Esc Anna Nery. 2018;22(4):e20170372. https://doi.org/10.1590/2177-9465-ean-2017-0372

11. Pereira LP, Nery AA. Planning, management and actions of men's health in the family health strategy. Esc Anna Nery. 2014;18(4):635-43. https://doi.org/10.5935/1414-8145.20140090

12. Brasil. Ministério da Saúde, Fundação Oswaldo Cruz, Instituto Nacional de Saúde da Mulher, da Criança e do Adolescente Fernandes Figueira. Fortalecimento da Política Nacional de Atenção Integral à Saúde do Homem (PNAISH): compromisso versus ação na atenção básica. Brasília, DF: Instituto Nacional de Saúde da Mulher, da Criança e do Adolescente Fernandes Figueira; 2013.

13. Lefevre F, Lefevre AM. Discourse of the collective subject: social representations and communication interventions. Texto Contexto Enferm. 2014;23(2):502-7. https://doi.org/10.1590/0104-07072014000000014 
14. Moreira RL, Fontes WD, Barboza TM. Difficulties of the man in primary healthcare: the speech of nurses. Esc Anna Nery. 2014;18(4):615-21. https://doi.org/10.5935/1414-8145.20140087

15. Mozer IT, Corrêa AC. Implementation of the National Policy for Men's Health: case in a Brazilian capital. Esc Anna Nery. 2014;18(4):578-85. https://doi.org/10.5935/1414-8145.20140082

16. Silveira CLG, Melo VFC, Barreto AJR. Attention to the health of men in primary health care: an integrative review. Rev Enferm UFPE On Line. 2017;11(supl 3):1528-35. https://doi.org/10.5205/1981-8963-v11i3a13998p1528-1535-2017

17. Moura MC, Soares CC, Lago EC, Batista MR, Oliveira RF, Rocha FC. [Human health situation to seek the unified health system services]. R Interd [Internet] 2017 [cited 2020 Apr 16];10(1):62-70. Portuguese. Available from: https://revistainterdisciplinar.uninovafapi.edu.br/ index.php/revinter/article/view/1016

18. Waling A, Fildes D. 'Don't fix what ain't broke': evaluating the effectiveness of a Men's Shed in inner-regional Australia. Health Soc Care Community. 2017;25(2):758-68. https://doi.org/10.1111/hsc.12365

19. Salgado, DM, Knowlton, AL, Johnson BL. Men's health-risk and protective behaviors: the effects of masculinity and masculine norms. Psychol Men Masc. 2019;(20):266-75. https://doi.org/10.1037/men0000211

20. Houman JJ, Eleswarapu SV, Mills JN. Current and future trends in men's health clinics. Transl Androl Urol. 2020;9(Suppl 2):S116-22. https://doi.org/10.21037/tau.2019.08.33

21. Brasil. Ministério da Saúde. Perfil da situação de saúde do homem no Brasil. Rio de Janeiro: Fundação Oswaldo Cruz; 2012.

22. Moura EC, Santos W, Neves AC, Gomes R. [Men's health care in the scope of the Family Health] Strategy. Cienc Saúde Coletiva. 2014;19(2):429-38. Portuguese. https://doi.org/10.1590/1413-81232014192.05802013

23. Leone JE, Rovito MJ, Mullin EM, Mohammed SD, Lee CS. Development and testing of a conceptual model regarding men's access to health care. Am J Mens Health. 2017;11(2):262-74. https://doi.org/10.1177/1557988316671637

24. Lovett D, Rasmussen B, Holden C, Livingston PM. Are nurses meeting the needs of men in primary care? Aust J Prim Health. 2017;23(4):319-22. https://doi.org/10.1071/PY16106

25. Santos KC, Fonseca DF, Oliveira PP, Duarte AG, Melo JM, Souza RS. [Men's health care: construction and validation of a tool for nursing consultation]. Rev Bras Enferm. 2020;73(3):e20190013. https://doi.org/10.1590/0034-7167-2019-0013

26. Storino LP, Souza KV, Silva KL. Men's health needs in primary care: user embracement and forming links with users as strengtheners of comprehensive health care. Esc Anna Nery. 2013;17(4):638-45. https://doi.org/10.5935/1414-8145.20130006

27. Leone JE, Rovito MJ, Mullin EM, Mohammed SD, Lee CS. Development and testing of a conceptual model regarding men's access to health care. Am J Men Health. 2017;11(2):262-74. https://doi.org/10.1177/1557988316671637

28. Modesto AA, Lima RL, D'Angelis AC, Augusto DK. [A not-so-blue November: debating screening of prostate cancer and men's health]. Interface (Botucatu). 2018;22(64):251-62. Portuguese. https://doi.org/10.1590/1807-57622016.0288

29. Sousa AR, Queiroz AM, Florencio RM, Portela PP, Fernandes JD, Pereira A. [Men on basic health attention services: repercussions of the social construction of masculinities]. Rev Baiana Enf. 2016;30(3):1-10. Portuguese. https://doi.org/10.18471/rbe.v30i3.16054

30. Silva JC, Nasif SS, Sousa AR, Santos SD, Mota TN, Pereira A. Clinical characteristics of men met in emergency care unit. Rev Baiana Enferm. 2020;34:e34702. https://doi.org/10.18471/rbe.v34.34702 